\title{
Rare and forbidden kaon decays at NA62
}

\author{
Monica Pepe ${ }^{1, a, b}$ \\ ${ }^{1}$ INFN Sezione di Perugia
}

\begin{abstract}
A precision lepton universality test by measurement of the helicity suppressed ratio of leptonic decay rates of the charged kaon with $\sim 150000 K^{ \pm} \rightarrow e^{ \pm} v$ decays collected by the NA62 experiment in 2007-08 is presented. The record accuracy of $0.4 \%$ constrains the parameter space of new physics models with extended Higgs sector, a fourth generation of quarks and leptons or sterile neutrinos.

An improved upper limit on the rate of the lepton number violating decay $K^{ \pm} \rightarrow \pi^{\mp} \mu^{ \pm} \mu^{ \pm}$, which probes the resonant enhancement of the rate in the presence of heavy Majorana neutrinos in the $\sim 100 \mathrm{MeV}$ range, is presented.

The rare decays $K^{+} \rightarrow \pi^{+} v \bar{v}$ are excellent processes to make tests of new physics at the highest scale complementary to LHC thanks to their theoretically cleaness. The NA62 experiment at CERN SPS aims to collect of the order of 100 events in two years of data taking, keeping the background at the level of $10 \%$.
\end{abstract}

\footnotetext{
ae-mail: monica.pepe@pg.infn.it

${ }^{b}$ On behalf of NA62 Collaboration: G. Aglieri Rinella, R. Aliberti, F. Ambrosino, B. Angelucci, A. Antonelli, G. Anzivino, R. Arcidiacono, I. Azhinenko, S. Balev, J. Bendotti, A. Biagioni, C. Biino, A. Bizzeti, T. Blazek, A. Blik, B. Bloch-Devaux, V. Bolotov, V. Bonaiuto, M. Bragadireanu, D. Britton, G. Britvich, N. Brook, F. Bucci, V. Buescher, F. Butin, E. Capitolo, C. Capoccia, T. Capussela, V. Carassiti, N. Cartiglia, A. Cassese, A. Catinaccio, A. Cecchetti, A. Ceccucci, P. Cenci, V. Cerny, C. Cerri, O. Chikilev, R. Ciaranfi, G. Collazuol, P. Cooke, P. Cooper, G. Corradi, E. Cortina Gil, F. Costantini, A. Cotta Ramusino, D. Coward, G. D’Agostini, J. Dainton, P. Dalpiaz, H. Danielsson, J. Degrange, N. De Simone, D. Di Filippo, L. Di Lella, N. Dixon, N. Doble, V. Duk, V. Elsha, J. Engelfried, T. Enik, V. Falaleev, R. Fantechi, L. Federici, M. Fiorini, J. Fry, A. Fucci, L. Fulton, S. Gallorini, L. Gatignon, A. Gianoli, S. Giudici, L. Glonti, A. Goncalves Martins, F. Gonnella, E. Goudzovski, R. Guida, E. Gushchin, F. Hahn, B. Hallgren, H. Heath, F. Herman, D. Hutchcroft, E. Iacopini, O. Jamet, P. Jarron, K. Kampf, J. Kaplon, V. Karjavin, V. Kekelidze, S. Kholodenko, G. Khoriauli, A. Khudyakov, Yu. Kiryushin, K. Kleinknecht, A. Kluge, M. Koval, V. Kozhuharov, M. Krivda, Y. Kudenko, J. Kunze, G. Lamanna, C. Lazzeroni, R. Leitner, R. Lenci, M. Lenti, E. Leonardi, P. Lichard, R. Lietava, L. Litov, D. Lomidze, A. Lonardo, N. Lurkin, D. Madigozhin, G. Maire, A. Makarov, I. Mannelli, G. Mannocchi, A. Mapelli, F. Marchetto, P. Massarotti, K. Massri, P. Matak, G. Mazza, E. Menichetti, M. Mirra, M. Misheva, N. Molokanova, J. Morant, M. Morel, M. Moulson, S. Movchan, D. Munday, M. Napolitano, F. Newson, A. Norton, M. Noy, G. Nuessle, V. Obraztsov, S. Padolski, R. Page, V. Palladino, A. Pardons, E. Pedreschi, M. Pepe, F. Perez Gomez, M. Perrin-Terrin, P. Petrov, F. Petrucci, R. Piandani, M. Piccini, D. Pietreanu, J. Pinzino, M. Pivanti, I. Polenkevich, I. Popov, Yu. Potrebenikov, D. Protopopescu, F. Raffaelli, M. Raggi, P. Riedler, A. Romano, P. Rubin, G. Ruggiero, V. Russo, V. Ryjov, A. Salamon, G. Salina, V. Samsonov, E. Santovetti, G. Saracino, F. Sargeni, S. Schifano, V. Semenov, A. Sergi, M. Serra, S. Shkarovskiy, A. Sotnikov, V. Sougonyaev, M. Sozzi, T. Spadaro, F. Spinella, R. Staley, M. Statera, P. Sutcliffe, N. Szilasi, D. Tagnani, M. Valdata-Nappi, P. Valente, M. Vasile, V. Vassilieva, B. Velghe, M. Veltri, S. Venditti, M. Vormstein, H. Wahl, R. Wanke, P. Wertelaers, A. Winhart, R. Winston, B. Wrona, O. Yushchenko, M. Zamkovsky, A. Zinchenko.
} 


\section{Introduction}

Kaon physics offers several opportunities for a deep study of the Standard Model (SM) investigating the flavour sector of fundamental interactions and being sensitive to possible effects of New Physics. NA62 and the NA48/2, NA48/1, NA48, NA31 series of experiments represent the long term CERN program in kaon physics: they are fixed target experiments at the CERN SPS dedicated to the study of $\mathrm{CP}$ violation and rare decays in the kaon sector with different beam configurations. While the NA48 experimental set-up has been upgraded in the course of operations, NA62 is a new experiment dedicated to the measurement of the ultra-rare $K^{+} \rightarrow \pi^{+} v \bar{v}$ decay.

\section{Test of Lepton Flavour Universality in $K^{ \pm} \rightarrow l^{ \pm} v$ decays}

The ratio $R_{k}=\Gamma\left(K_{e 2}\right) / \Gamma\left(K_{\mu 2}\right)$ of kaon leptonic decay rates is computed with very high accuracy in the SM thanks to cancellation of hadronic effects: the SM prediction inclusive of Internal Bremsstrahlung (IB) radiation is [1]:

$$
R_{K}^{S M}=\frac{m_{e}^{2}}{m_{\mu}^{2}} \cdot\left(\frac{m_{K}^{2}-m_{e}^{2}}{m_{K}^{2}-m_{\mu}^{2}}\right)^{2} \cdot\left(1+\delta R_{Q E D}\right)=(2.477 \pm 0.001) \cdot 10^{-5}
$$

where $\delta R_{Q E D}=(3.79 \pm 0.04) \%$ is a correction due to the IB part of the radiative decay $K^{ \pm} \rightarrow e^{ \pm} v \gamma$ included in the $R_{K}$ definition. The $K_{e 2}$ mode is strongly helicity suppressed, as taken into account by the factor $\frac{m_{e}^{2}}{m_{\mu}^{2}}$, thus the amplitude is sensitive to contributions from physics beyond the SM. Within extensions of the SM involving two Higgs doublets, $R_{K}$ is sensitive to lepton flavour violating effects induced by loop processes with the charged Higgs boson $\left(H^{ \pm}\right)$exchange [2, 3]. A recent study [4] reported about the $R_{K}$ enhancement in the percent range within the Minimal Supersymmetric Standard Model (MSSM). The most precise measurements of $R_{K}$ were performed recently by the KLOE [5] and NA62 [6] experiments. In this paper the improved NA62 [7] result based on data collected in 2007 and 2008 is presented.

\subsection{Beam and Detector}

The beam line of the NA48/2 [8] experiment was used for the NA62 $R k$ measurement: positive and negative hadron beams were produced in the same beryllium target by impinging $400 \mathrm{GeV}$ protons from the CERN/SPS accelerator. The beam momentum range of $(74 \pm 1.4) \mathrm{GeV} / \mathrm{c}$ was selected by two front-end achromats and focused $\sim 200 \mathrm{~m}$ downstream, at the end of the decay region.

Charged decay product momenta were measured by a magnetic spectrometer consisting of two sets of drift chambers and a central dipole magnet, providing a momentum resolution $\sigma(p) / p=0.48 \% \oplus$ $0.009 \% \cdot p(\mathrm{p}$ in $\mathrm{GeV} / \mathrm{c})$. A hodoscope consisting of two planes of plastic scintillators segmented into horizontal and vertical strips provided a fast trigger for charged particles with very good ( $\sim 150 \mathrm{ps})$ time resolution. A liquid Krypton calorimeter $(\mathrm{LKr}), 27 X_{0}$ deep, used to measure electromagnetic energy deposition of photons and electrons with a resolution $\sigma_{E} / E=3.2 \% \mathrm{GeV}^{1 / 2} / \sqrt{E} \oplus 9 \% / E \oplus$ $0.42 \%$ (E in $\mathrm{GeV}$ ), was used for lepton identification and photon rejection in the present analysis. The detector is described in more detail in Ref.[9].

\subsection{The measurement of $R_{K}$}

This measurement is based on a sample of $2 \times 10^{10} K^{ \pm}$decays collected in 2007-2008 at the CERN SPS. $K_{e 2}$ and $K_{\mu 2}$ decays were collected simultaneously, such that the measurement is independent of 
the kaon flux and many systematic effects cancel at first order. Given the dependence of background and acceptance on momentum, the $R_{K}$ measurement is performed in 10 bins of lepton momentum between 14 and $65 \mathrm{GeV} / \mathrm{c}$ :

$$
R_{K}=\frac{1}{D} \cdot \frac{N\left(K_{e 2}\right)-N_{B G}\left(K_{e 2}\right)}{N\left(K_{\mu 2}\right)-N_{B G}\left(K_{\mu 2}\right)} \cdot \frac{A\left(K_{\mu 2}\right) \cdot f_{\mu} \cdot \epsilon\left(K_{\mu 2}\right)}{A\left(K_{e 2}\right) \cdot f_{e} \cdot \epsilon\left(K_{e 2}\right)} \cdot \frac{1}{f_{L K r}}
$$

where $N\left(K_{l 2}\right)$ are the numbers of selected $K_{l 2}$ candidates $(l=e, \mu), N_{B G}\left(K_{l 2}\right)$ the numbers of background events, $\mathrm{A}\left(K_{l 2}\right)$ the geometrical acceptances, $f_{l}$ the lepton identification efficiencies, $\epsilon\left(K_{l 2}\right)$ the trigger efficiencies, $f_{L K r}$ is the electromagnetic calorimeter $(\mathrm{LKr})$ global read out efficiency and $\mathrm{D}=150$ is the downscaling factor of the $K_{\mu 2}$ trigger.

A common selection is applied given the similar topology of the two $K_{l 2}$ decays. They are then kinematically separated by reconstructing the squared missing mass $M_{\text {miss }}^{2}(l)=\left(P_{K}-P_{l}\right)^{2}$ (where $P_{K}$ and $P_{l}$ are the kaon and lepton four-momenta) assuming that the charged track is an electron or a muon. Lepton identification is based on the ratio $E / p$ of the energy deposited in the LKr calorimeter to the momentum measured in the spectrometer. Electrons and positrons are identified requiring $(E / p)_{\min }<E / p<1.1$ (where $(E / p)_{\min }$ is 0.95 for $\mathrm{p}>25 \mathrm{GeV} / \mathrm{c}$ and 0.9 otherwise), while charged particles with $E / p<0.85$ are identified as muons.

Figure 1 shows the $M_{m i s s}^{2}$ spectra for $K_{e 2}$ (on the left) and $K_{\mu 2}$ (on the right) candidates. The distributions of both $K_{l 2}$ candidates are compared to the sum of normalized estimated signal and background components.
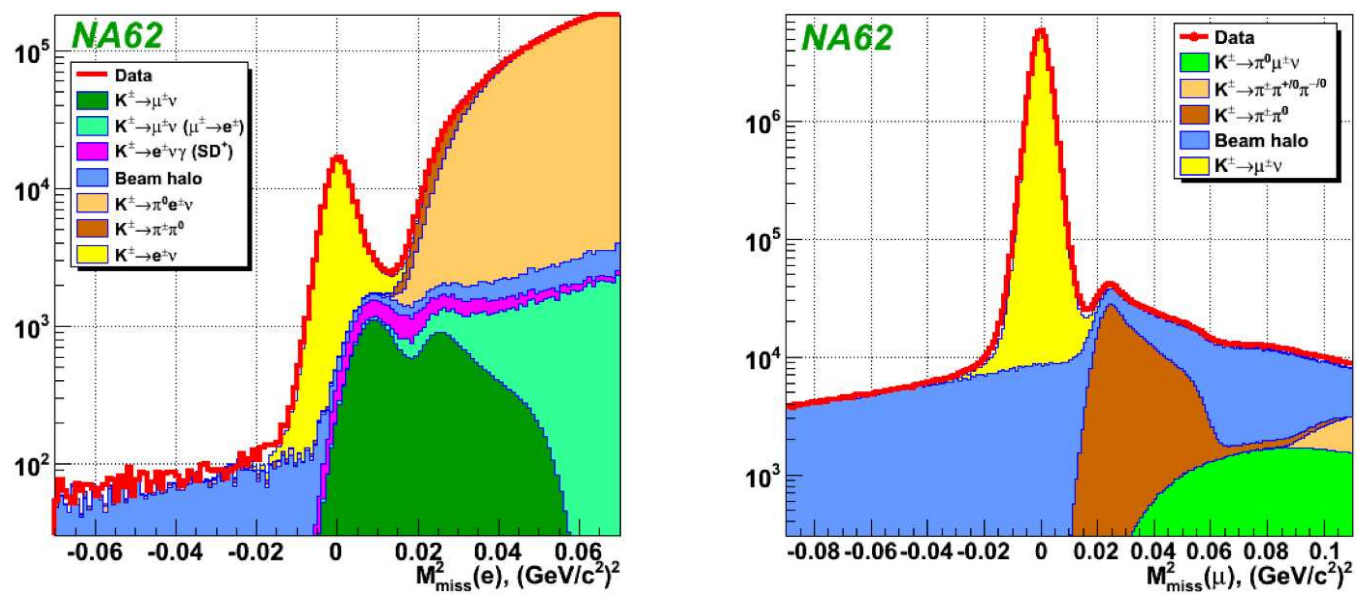

Figure 1. Reconstructed $M_{\text {miss }}^{2}$ distributions of $K_{e 2}$ (left) and $K_{\mu 2}$ (right) candidates compared to the sums of estimated signal and background components.

The main source of background to the $K_{e 2}$ sample arises from beam halo muons and $K_{\mu 2}$ decays with a misidentified muon, mainly due to catastrophic bremsstrahlung with consequent high energy deposit in the $\mathrm{LKr}$ calorimeter. The misidentification probability has been precisely measured directly from data, comparing a muon enriched sample acquired with a lead wall $\sim 9 X_{0}$ thick installed in front of the calorimeter with a partial geometric acceptance. Other background sources come from $K^{ \pm} \rightarrow e^{ \pm} v \gamma$ decays with the gamma lost, and $K^{ \pm} \rightarrow \pi^{0} e^{ \pm} v$ and $K^{ \pm} \rightarrow \pi^{ \pm} \pi^{0}$ decays with pion misidentification. The $K_{\mu 2}$ sample is almost background free. 
NA62 selected $145958 K_{e 2}$ candidates with a background contamination of $(10.95 \pm 0.27) \%$ : this is the largest $K_{e 2}$ sample ever collected. The result, obtained performing a $\chi^{2}$ fit to 40 statistically independent measurements of $R_{K}$ taking into account correlations of the systematic uncertainties, is:

$$
R_{K}=\left(2.488 \pm 0.007_{\text {stat }} \pm 0.007_{\text {syst }}\right) \times 10^{-5}=(2.488 \pm 0.010) \times 10^{-5}
$$

The stability of the measurement as a function of the lepton momentum is shown in figure 2.

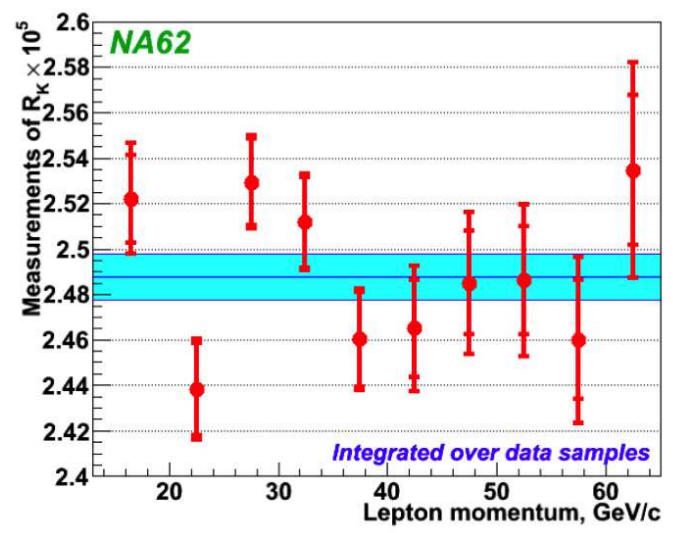

Figure 2. $R_{K}$ as a function of the lepton momentum: statistical and total errors are shown on individual points. The horizontal lines and bands indicates the $\chi^{2}$ fit result with combined statistical and systematic uncertainties.

Details of the data analysis can be found in [7]. This is the most precise measurement of $R_{K}$ to date and is consistent with earlier measurements and with the SM expectations. The experimental uncertainty on $R_{K}$ is still an order of magnitude larger than the uncertainty on the SM prediction, which motivates further measurements at improved precision.

\section{The measurement of the $K^{+} \rightarrow \pi^{+} v \bar{v}$ decay}

The $K^{+} \rightarrow \pi^{+} v \bar{v}$ decay, together with $K_{L} \rightarrow \pi^{0} v \bar{v}$, is a decisive test of the Standard Model sensitive to new physics. These decays are FCNC processes strongly suppressed in SM, dominated by short-distance dynamics and top quark loop contributions. The amplitude is governed by one single semileptonic operator which hadronic matrix element can be parameterized in terms of the well known experimentally $K^{+} \rightarrow \pi^{0} e^{+} v$ branching ratio, allowing extremely precise theoretical predictions. The SM prediction [10] for the $K^{+} \rightarrow \pi^{+} v \bar{v}$ channel is $B R=\left(7.81_{-0.71}^{+0.80} \pm 0.29\right) \times 10^{-11}$, where the uncertainty is dominated by the error on the CKM matrix elements and the irreducible theoretical uncertainties are at $1 \%$ level. The extreme theoretical cleanness of these decays remains also in many new physics scenarios $[11,12]$.

The existing measurement of the $K^{+} \rightarrow \pi^{+} v \bar{v}$ branching ratio $B R=1.73_{-1.05}^{+1.15} \times 10^{-10}$, based on $7 K^{+} \rightarrow \pi^{+} v \bar{v}$ candidates observed by the BNL E787/E949 experiments [13], is still consistent with SM expectations within errors with a central value about twice larger, however at least a $10 \%$ accuracy measurement is required in order to provide a significative test of the SM and of possible new scenarios. Furthermore, the precise measurement of $K^{+} \rightarrow \pi^{+} v \bar{v}$ will provide an alternative measurement of $V_{t d}$ with respect to B decays.

\subsection{The NA62 experiment}

A new beam line from the CERN SPS $400 \mathrm{GeV} / \mathrm{c}$ primary proton beam impinging on a beryllium target will produce an intense secondary hadron beam of positive charge with a central momentum 
of $75 \mathrm{GeV} / \mathrm{c}$ and a momentum bite of $\sim 1 \%$, corresponding to an average rate of about $750 \mathrm{MHz}$. The beam contains $6 \%$ of $K^{+}$and is almost positron free. This beam line will provide $4.5 \cdot 10^{12} \mathrm{~K}^{+}$ decays/year. The layout of the experimental set-up is schematically shown in figure 3

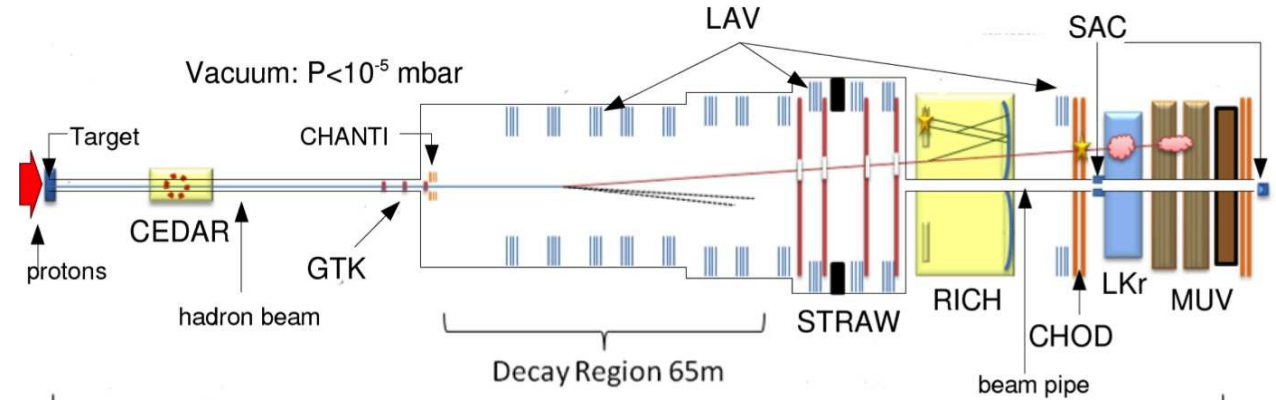

Figure 3. The NA62 experiment layout.

Identification of kaons in the unseparated beam is done by a differential Cherenkov counter (KTAG) with an efficiency of at least $95 \%$ and a time resolution of about 100 ps. A beam spectrometer (Gigatracker, GTK), consisting of three silicon pixel stations exposed to an instantaneous rate of $\sim 750$ $\mathrm{MHz}$, will measure time, coordinates and momentum of individual particles with a beam momentum resolution of $0.2 \%$ and a time resolution of $\sim 200$ ps per station. The direction and the momentum of secondary charged particles will be measured by the straw spectrometer (STRAW) consisting of four chambers and a high aperture dipole magnet, providing a momentum resolution of $\sigma(p) / p \sim 0.32 \% \oplus 0.008 \% \times p(\mathrm{GeV} / \mathrm{c})$. The Photon Veto system will provide $\pi^{0}$ rejection for the $\pi^{+} \pi^{0}$ background: it comprises several detectors reaching a quasi-hermetic coverage below $1 \mathrm{mrad}$. The Large Angle Veto (LAV) surrounding the decay region consists of lead glass blocks arranged in 12 annular rings. The maximum tolerable inefficiency in the LAV detectors for photons with energies as low as $200 \mathrm{MeV}$ is $10^{-4}$. Photons in the intermediate angular region are detected by the NA48 Liquid Krypton Electromagnetic calorimeter (LKr). Two small shashlik calorimeters act as photon vetoes at very small angles: the Intermediate Ring Calorimeter (IRC) covering the region around the inner radius of the $\mathrm{LKr}$ and the Small Angle Calorimeter (SAC), covering the angular region down to zero degree. The kinematics of $\pi^{+} \pi^{0}$ decays is such that a low energy photon at large angle (more difficult to detect) is paired with a high energy one in the LKr or small angle calorimeters where high efficiency is expected. A $18 \mathrm{~m}$ long RICH detector filled with Neon at atmospheric pressure will provide $\pi-\mu$ separation in the momentum range $15-35 \mathrm{GeV} / \mathrm{c}$ with a rejection factor better than $5 \times 10^{-3}$ and the event time measurement with a resolution better than $100 \mathrm{ps,} \mathrm{minimizing} \mathrm{wrong} \mathrm{matching}$ with the parent particle measured by the KTAG. The detector is completed by a Charged Hodoscope (CHOD) providing fast track measurements for the trigger, a veto for charged particles at small angles (CHANTI) that will reduce background induced by inelastic interactions of the beam with the collimator and the GTK, and a Muon Detector (MUV) placed at the end of the apparatus after an iron wall. The detailed description of the NA62 detectors can be found in [14].

\subsection{Background rejection and sensitivity}

The goal of the NA62 experiment is to collect about $100 K^{+} \rightarrow \pi^{+} v \bar{v}$ events in two years of data taking keeping a $10 \%$ background contamination: this can be achieved using a decay in flight technique and 
a high intensity kaon beam able to provide $\sim 10^{13}$ kaon decays in the fiducial volume, assuming a $10 \%$ signal acceptance. The required small systematic uncertainty demands a background suppression factor of $\sim 10^{12}$. The experimental signature of a $K^{+} \rightarrow \pi^{+} v \bar{v}$ decay consists of only one positive track reconstructed in the downstream detectors matching with the $K^{+}$track in the beam. The presence of two undetectable neutrinos in the final state requires redundant measurement of the event kinematics. Backgrounds come from all the $K^{+}$decay modes, the most frequent ones are listed in Table 1 .

Table 1. BR of the most frequent $K^{+}$decays.

\begin{tabular}{ll}
\hline Decay mode & Branching Ratio \\
\hline$K^{+} \rightarrow \mu^{+} v$ & $63.5 \%$ \\
$K^{+} \rightarrow \pi^{+} \pi^{0}$ & $20.7 \%$ \\
$K^{+} \rightarrow \pi^{+} \pi^{+} \pi^{-}$ & $5.6 \%$ \\
$K^{+} \rightarrow \pi^{0} e^{+} v$ & $5.1 \%$ \\
$K^{+} \rightarrow \pi^{0} \mu \nu$ & $3.3 \%$ \\
$K^{+} \rightarrow \pi^{+} \pi^{0} \pi^{0}$ & $1.8 \%$ \\
\hline
\end{tabular}

The squared missing mass, defined as $m_{\text {miss }}^{2}=\left(P_{K}-P_{\pi}\right)^{2}$ (where $P_{K}$ and $P_{\pi}$ are the kaon and charged pion four momenta) assuming that the final charged particle is a pion, fully describes the decay kinematics and allows kinematical separation between signal and $92 \%$ of total background.
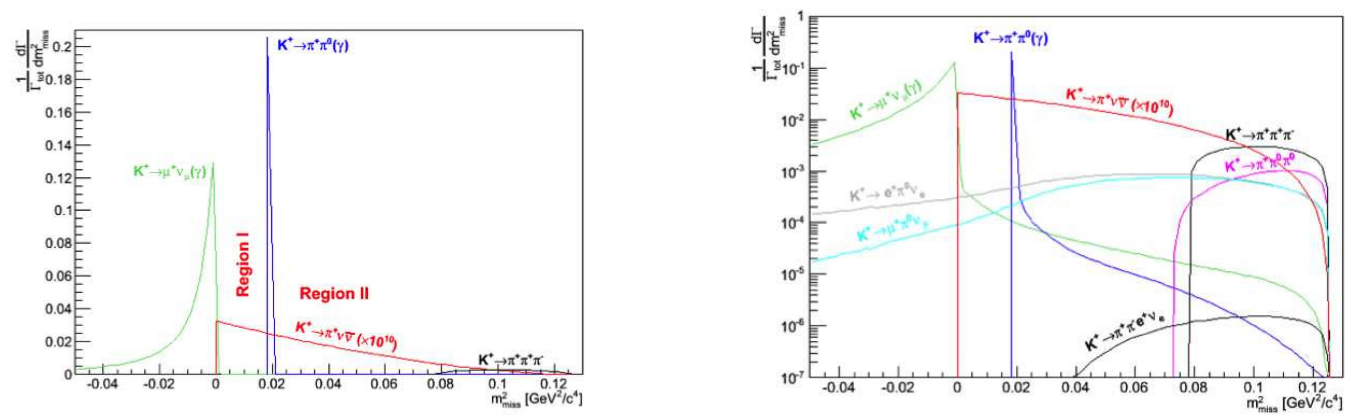

Figure 4. The $m_{m i s s}^{2}$ distributions: signal region definition for kinematically constrained background (left) and background not constrained by kinematics (right). Backgrounds are normalized according to their branching ratios; the signal is multiplied by a factor $10^{10}$.

As shown in figure 4 left, the backgrounds with largest branching ratios due to $K^{+} \rightarrow \pi^{+} \pi^{0}$ and $K^{ \pm} \rightarrow \mu^{ \pm} v$ decays define two signal regions around the $\pi^{0}$ peak where background enters only because of non gaussian tails in the squared missing mass resolution. The remaining $8 \%$ background (shown in figure 4 right) is not kinematically constrained and its rejection must rely on the veto system and particle identification (PID). The detector components give redundancy both in kinematics reconstruction and particle identification allowing background estimation directly from the data. GTK information will be combined with the STRAW spectrometer one to reconstruct the event and reject background with kinematics; precising timing will allow to associate the decay pion to the parent kaon; the PID system will be used to identify the Kaon in the charged beam (KTAG) and to separate pions from muons and electrons in the final state $(\mathrm{RICH})$; the efficient veto systems will reject events with photons and muons. 
Sensitivity studies, for kaon decays taking place in the first $60 \mathrm{~m}$ of the decay volume and for reconstructed downstream pion momentum between 15 and $35 \mathrm{GeV} / \mathrm{c}$, indicate that 45 events/year are expected with a total background of less than $20 \%$. Part of the experimental apparatus has been commissioned during a technical run in 2012. The experiment started the first physics run at lower beam intensity in October 2014 and the run at nominal beam intensity will take place in 2015 to 2017.

\section{The measurement of $K^{ \pm} \rightarrow \pi^{\mp} \mu^{ \pm} \mu^{ \pm}$}

Lepton Flavour (LFV) and Lepton Number Violation (LNV) processes are good probes having unmeasurable rates in SM. Kaons are historically competitive in searches for LFV and LNV phenomena : the abundant production provides high statistics, simple topologies give clean signatures, they have controllable backgrounds. This brings sensitivity to very low branching ratios.

The LNV decay $K^{ \pm} \rightarrow \pi^{\mp} \mu^{ \pm} \mu^{ \pm}$is forbidden in SM, however it can proceed in new physics models via a Majorana neutrino exchange [15]. This observation would probe the resonant enhancement of the rate in the presence of heavy Majorana neutrinos in the $100 \mathrm{MeV}$ mass range and upper limits on its rate would provide the strongest constraint on the effective Majorana neutrino mass.

The NA48/2 experiment performed a search for this decay using data collected in 2003 and 2004 with simultaneous $K^{+} / K^{-}$beams.
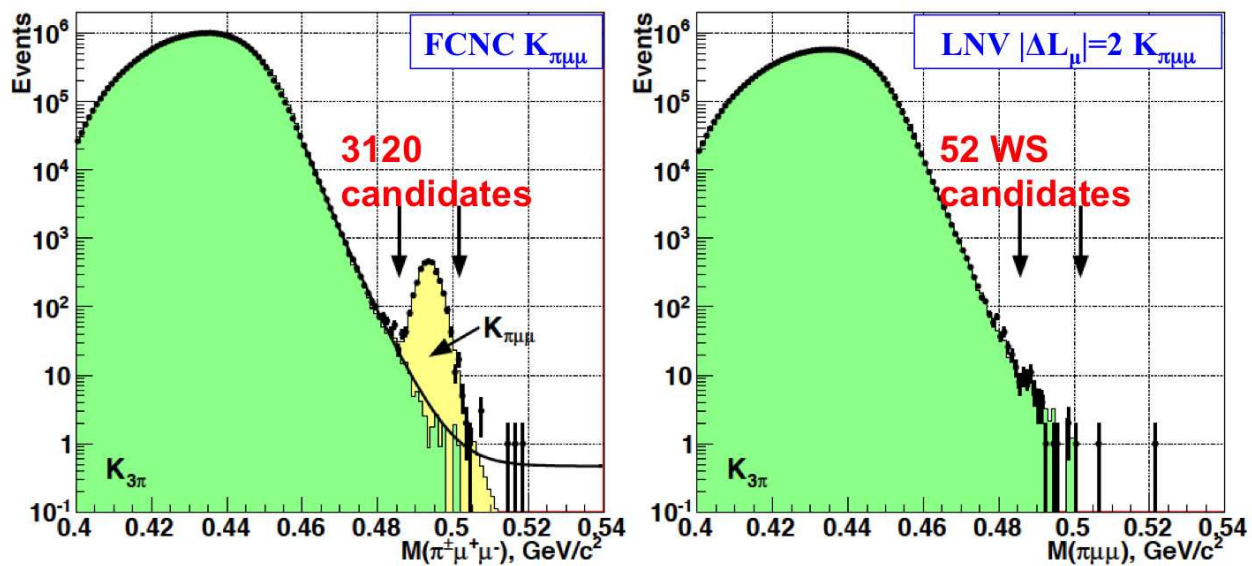

Figure 5. Reconstructed $M_{\pi \mu \mu}$ spectra for $K_{\pi \mu \mu}$ correct sign (left) and wrong sign (right) candidates: dots are data, filled area are $K_{3 \pi}$ and $K_{\pi \mu \mu}$ simulations. The arrows indicate the signal region.

A sample of $3120 K^{ \pm} \rightarrow \pi^{ \pm} \mu^{ \pm} \mu^{\mp}$ FCNC candidates was collected allowing a detailed study of the decay properties. Their reconstructed invariant mass spectrum is shown in figure 5 left. Figure 5 right shows the spectrum for events with two leptons of the same sign (wrong sign) in the final state, corresponding to LNV violating decays: 52 wrong sign events fall in the signal region where $52.6 \pm 19.8$ background events are expected from a MC simulation. This translates into an upper limit $B R\left(K^{ \pm} \rightarrow \pi^{\mp} \mu^{ \pm} \mu^{ \pm}\right)<1.1 \times 10^{-9}$ at $90 \%$ CL [16], improving by almost a factor of 3 the best previous limit [17] and allowing a bound on the effective Majorana neutrino mass of $\left\langle m_{\mu \mu}\right\rangle \lesssim 300 \mathrm{GeV} / \mathrm{c}^{2}$ to be established [18]. The present upper limit can be further improved by the NA62 experiment that will collect $\sim 10^{13}$ kaon decays reaching a single event sensitivity $(\mathrm{SES}) \sim 10^{-12}$. 


\section{References}

[1] V. Cirigliano and I. Rosell, JHEP 10, 005 (2007)

[2] A. Masiero, P. Paradisi, R. Petronzio, Phys.Rev. D74, 011701 (2006)

[3] A. Masiero, P. Paradisi, R. Petronzio, JHEP 0811, 042 (2008)

[4] J. Girrbach and U. Nierste, arXiv:1202.4906 (2012)

[5] F. Ambrosino et al., Eur. Phys. J. C64, 627 (2009)

[6] C. Lazzeroni et al., Phys. Lett. B698, 105 (2011)

[7] C. Lazzeroni et al., Phys. Lett. B719, 326 (2013)

[8] R. Batley et al., Eur. Phys. J. C52, 875 (2007)

[9] V. Fanti et al., Nucl.Instrum.Meth. A574, 433 (2007)

[10] J. Brod, M. Gorbahn and E. Stamou, Phys.Rev. D83, 034030 (2011)

[11] G. Isidori et al., arXiv:0604074 [hep-ph], JHEP 0608, 064 (2006)

[12] M. Blanke et al., arXiv:0906.5454 [hep-ph], Acta Phys. Polon. B41, 657 (2010)

[13] A. V. Artamonov et al., Phys. Rev. Lett. 101, 191802 (2008)

[14] The NA62 Collaboration, Technical Design, NA62-10-07, http://cds.cern.ch/record/1404985

[15] A. Atre, T. Han, S. Pascoli and B. Zhang, JHEP 0905, 030 (2009)

[16] J. R. Batley et al. Phys. Lett. B697, 107 (2011)

[17] R. Appel et al., Phys. Rev. Lett. 85, 2877 (2000)

[18] K. Zuber, Phys. Lett. B479, 33 (2000). 